\title{
PROMOTING PERSONALIZED LEARNING DESIGN: THE ROLE OF ONLINE PEDAGOGICAL INTERVENTION
}

\author{
Hamdy A. Abdelaziz, Hamdan Bin Mohammed Smart University, United Arab Emirates
}

\section{Abstract}

Online learning technology and design has maximized and optimized the potential chances of personalized, customized, and adaptive learning. This theoretical paper is proposing a new dynamic pedagogical intervention model for effective personalized learning design. The author is trying to share a personal and practical answer to the following two questions: (a) What are the disruptive learning principles of the third renaissance learning paradigm that impact pedagogical engineering and intervention for personalized learning design? (b) What is the suggested model for effective online pedagogical intervention to promote personalized learning design? This perspective was guided by ten emergent disruptive learning principles of the third renaissance learning paradigm that impact online pedagogical engineering, management and intervention for personalized learning design. Effective online pedagogical intervention has four major dimensions that are grounded/interacted and focused on four metaphoric lenses: (a) types of learners (4Cs): Casual, Committed, Concentrated and Continuing; (b) pedagogical levels (4Ps): Intelligent, Agile, Distributed and Situated Pedagogy; (c) intervention levels (4Es): Enriching, Enhancing, Engaging and Empowering; and (d) online assessment frames (4As): Assessment of learning, Assessment for learning, Assessment as learning, and Assessment in learning.

\section{Introduction}

Online educational systems and programs worldwide are facing many challenges and issues such as: retention rate, reduce time-to-degree, non-formal and open-personalize learning, freeload and overload learners, pedagogical distance (isolation), organizational analytics and quality matter - to mention few. These challenges are pushing to reshape and re-engineering online pedagogical design models and practices to promote the competencies of personalized learning design.

Since the beginning of the $21^{\text {st }}$ century, there are major shifts in educational paradigm. These shifts are applied on both conventional and online learning contexts. Of these shifts: (a) from standardization to personalization, (b) from content engagement to knowledge engagement and creation, (c) from judgmental assessment to developmental and dynamic assessment, (d) from dominant culture of testing to dominant culture of value and learnability, (e) from instructional systems design (ISD) to personalized learning design (PLD), and f) from problem solving to design thinking and design authoring (Abdelaziz, 2015). 
In the third renaissance-learning paradigm, new advances in the science of learning and emerging education goals emphasize the importance of helping people control, mediate and regulate their own personalized learning. Therefore, the roles of online pedagogy should be expanded beyond the traditional concepts of delivering and testing to help learners build their own personalized values and illustrate their deep cognition to themselves, peers, teachers, their macro and micro community and professional networks (Abdelaziz, 2014a). The ultimate goal of any pedagogical and educational system is to prepare learners to become knowledge generators and life-long learners (Ally, 2008). Without a plan for transformative pedagogical design, smart technology often remains ancillary to personalized learning.

In online learning contexts, the role of pedagogical design became an integral part of effective personalized learning, especially with increased demand on MOOCs, cMOOCs, and on online learning certificates, degrees, and programs. As such, pedagogical design models are also changed to play a multi-dimension role. According to Gustafson \& Branch (2002), the role of models in instructional development is to provide us with conceptual and communication tools that we can use to: (a) visualize, direct, and manage processes for generating episodes of guided learning; (b) view both the linear and concurrent aspects of instructional development; and (c) select appropriate operational tools to maximize learning paradigms.

With the increasing demand of online courses, higher education institutions started to offer open and online courses and programs. Massive Open Online Courses (MOOCS) are example of unlimited participation and open access course via the Web. Although, these courses have varieties of electronic content, e.g. videos, lectures, and readings, but still have an issue regarding the pedagogical design and management. To activate online learners and increase their potential learning abilities and personalized and professional outcomes, there should be online pedagogical intervention initiatives and practical models and approaches.

This theoretical paper is proposing a new dynamic pedagogical intervention model for effective personalized learning design. The researcher is trying to share a personal and practical answer to the following two questions:

- What are the disruptive learning principles of the third renaissance learning paradigm that impact pedagogical engineering for personalized learning design?

- What is the suggested model for effective online pedagogical intervention to promote personalized learning design?

\section{Pedagogical Intervention (PI)}

In online learning context, pedagogical intervention (PI) emphasizes on coaching, facilitating, and promoting learners' current and potential intellectual capabilities, dispositions and attributes. The purposes of PI are to know how and when online learners learn, unlearn, relearn and help others to learn collaboratively. The core idea and concern of pedagogical intervention is that it exceeds the teaching and social presence dimensions. Teaching and 
social presence are important but are not enough to reflect or promote online learners' cognitive tempo, epistemological belief and ontological identity or pattern.

Pedagogical intervention is also a tetrad process to help both online facilitators and learners to visualize, personalize, manage and capitalize online teaching and learning activities and feedback. The ultimate goal of pedagogical intervention is to re-engineer online learning context to be customized and dynamic to fit with each individual learner's needs and online groups' collective minds. It gives a great chance and support for online facilitators to transform their foci from content benchmarks to mental benchmarks. It is a dynamic process to re-engineering online learning context to achieve smart technologies integration in the delivery and production of knowledge, skills and competencies.

Online pedagogical intervention has four major lenses that reflect levels of learners, pedagogical levels, levels of intervention, and assessment frames. Pedagogical intervention in online teaching and learning context is a cubic and grounded/interacted perspective and focuses on four metaphoric lenses: (a) types of learners (4Cs): Casual, Committed, Concentrated and Continuing (Al-Awar, 2009); (b) pedagogical levels (4Ps): Intelligent, Agile, Distributed and Situated Pedagogy (Guardia et al., 2016); (c) intervention levels (4Es): Enriching, Enhancing, Engaging and Empowering (Abdelaziz, 2015), and (d) online assessment frames (4As): Assessment of learning, Assessment for learning, Assessment as learning, and Assessment in learning. These four dimensions are interacting vertically and horizontally to produce 256 techniques for effective personalized learning design. Figure 1 presents the pedagogical intervention dimensions/lenses.

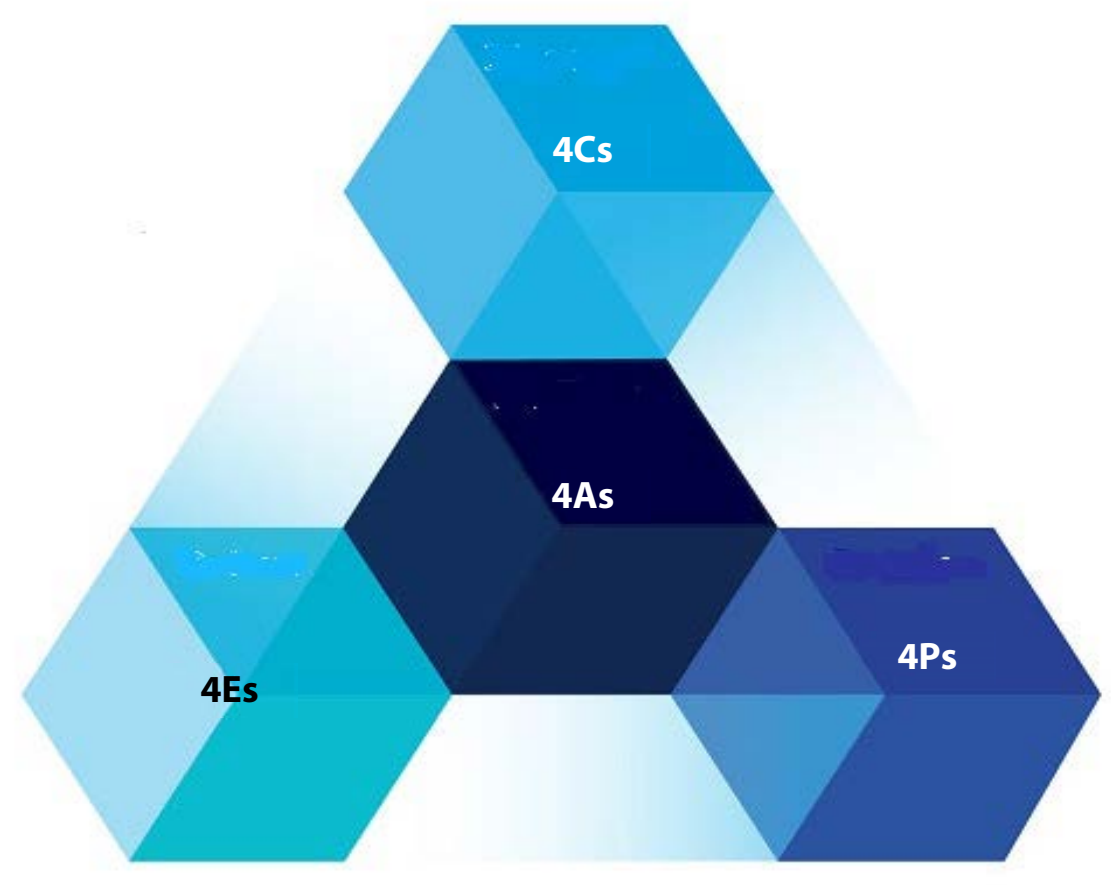

Figure 1. Pedagogical Intervention Dimensions/Lenses 


\section{Pedagogical Engineering (PE)}

Pedagogical engineering refers to all efforts that online facilitator takes into account to innovate, disrupt, customize, personalize and manage online teaching and learning smart solutions and applications that reflect online learners' level, epistemological bases of the content knowledge, and core values of learning. Pedagogical engineering is a paradigm of mental and psychological practice to activate and empower online learning programs and courses. The ultimate goal of pedagogical engineering is to manage and lead effective online courses delivery and assessment process by supporting online learners to explore, discover, generate and capitalize new academic and professional content knowledge and competencies. $\mathrm{PE}$ could also be used as transformative mindtool to design thinking, modelling, re-modelling and design authoring of online learning practices and experience.

\section{Grounded Goals of PI and PE}

PI and PE of online teaching and learning are guided by the following grounded/emergent goals:

- Build models and patterns of learning in the third renaissance age.

- Empower design thinking and design authoring among online learners.

- Create a culture of creativity and innovation in online learning context.

- Activate personalization and self-organized learning competencies among online learners.

- Activate knowledge synergy and economy.

- Activate ontological conceptions and pedagogical analytics in online learning context.

What are the disruptive learning principles of the third renaissance learning paradigm that impact pedagogical intervention and engineering for personalized learning design?

To answer previously stated question, the authors reviewed some of recently published models of online pedagogical design and its related issues, variables and applications (e.g. Abdelaziz, 2012; 2013a; 2013b; 2014a; 2014b; Anderson, 2008; Burke, \& Crozier, 2013; Chonody, 2015; Guardia, Witthaus, Rodriguez, \& Campillo, 2016; Lockyer, Heathcote, \& Dawson, 2013; Mekala, Shantha, \& Ponmani, 2017; Ramdass \& Masithulela, 2016; Robinson, 2011; Tegos, Demetriadis, Papadopoulos, \& Weinberger, 2016; Saville, Zinn, \& Elliott, 2005; Shum \& Ferguson, 2015; Silva, 2013; Westberry \& Fanken, 2015; Wise, 2014; Wright \& Collins, 2007; Zhu, Yu, \& Riezebos, 2016).

Pedagogical intervention and engineering paradigm is guided disruptive learning principles to suits online learning context (people, place and purpose). These principles are:

- Strong and profound online knowledge is gained through a networking process of collective mind and collective efficacy.

- Online cognition is an engineering process that needs immersive, interactive, active and reflective online mediator. 
- The wisdom of practice in online teaching and learning context is a continuous process of reflection-on-action not just reflection-in-action.

- Online teaching is a productive and developmental dialogue to reduce alternative conception and free-load achievement through well-design cognitive and affective scaffolds.

- Delivering effective online courses needs a transformative pedagogical design to empower online learners through tetrad-like and loop-like learning activates and feedback.

- Online cognition catalyst is enhanced through effective integration of communication tools.

- Effective pedagogical intervention emphasizes problem analysis and design thinking.

- Early vision of pedagogical intervention comes from analysing learners' cognitive and affective tempo.

- In online learning context, when and who have the same importance like what, why, how and where.

- Effective pedagogical design gives a considerable attention to learners' disposition. Current generation of learners are divers, dynamic and differentiated by nature.

\section{Pedagogical Intervention Techniques}

Most of online teaching programs and courses are delivering online materials and content in a linear manner such as online lecturers, video segments, readings, asynchronous and synchronous activities, and online assignments. This linearity may improve the quality control of online course delivery, but it does not improve and assure the quality and the impact of online learning (Abdelaziz, 2012). Online pedagogical intervention exceeds the traditional techniques of delivering online courses. It emphasizes multimodality techniques that increase the level interaction and interactivities in online learning context for any type of learners (regular and special needs learners). PI depends on techniques that decrease the level of isolation and free-load learning. PI techniques increase the level of engaging of online learner to produce, generate, and innovate new knowledge or solution. Pi techniques are new windows of immersive learning design that support design thinking and design authoring tools and skills. Of these techniques:

- Cognitive scaffolding.

- Ongoing feedback and feedforward (360-Degree).

- Productive dialogues (error - reduction techniques).

- Cognitive Guided Instruction (accuracy mirrors).

- $\quad$ PSI attributes (Triangulation Practices: TP, SP and CP).

- Loop Learning Activities (Tetrad-Like Activities).

- DA (Dynamic Assessment Techniques).

- Interteaching (Mutually Probing Conversation).

The authors believe that these grounded online pedagogical intervention techniques may help in increasing retention rate and reduce-time to degree. They may also close the gap between 
pedagogical distance (Isolation) and pedagogical presence; and the gap between free-load and over load learner in online learning context. In addition, these techniques may increase the level of learning and organizational analytics.

\section{What is the suggested model for effective online pedagogical intervention?}

To design this PI model, the researcher reviewed some of recently published models of online pedagogical design and its related applications in online learning context. PI is a cubic and grounded perspective and focuses on four integrated dimensions: (a) types of online learners (Al-Awar, 2009), (b) pedagogical patterns (Guardia et al., 2016), and (c) intervention levels (Abdelaziz, 2015), (d) online assessment frames. These four lenses are generally articulated in Figure 1. These four dimensions are integrated and dynamic in a specific manner in which it produces 256 techniques for effective online personalized learning design. Figures 2, 3, 4, and 5 represent each dimension and its related concepts.

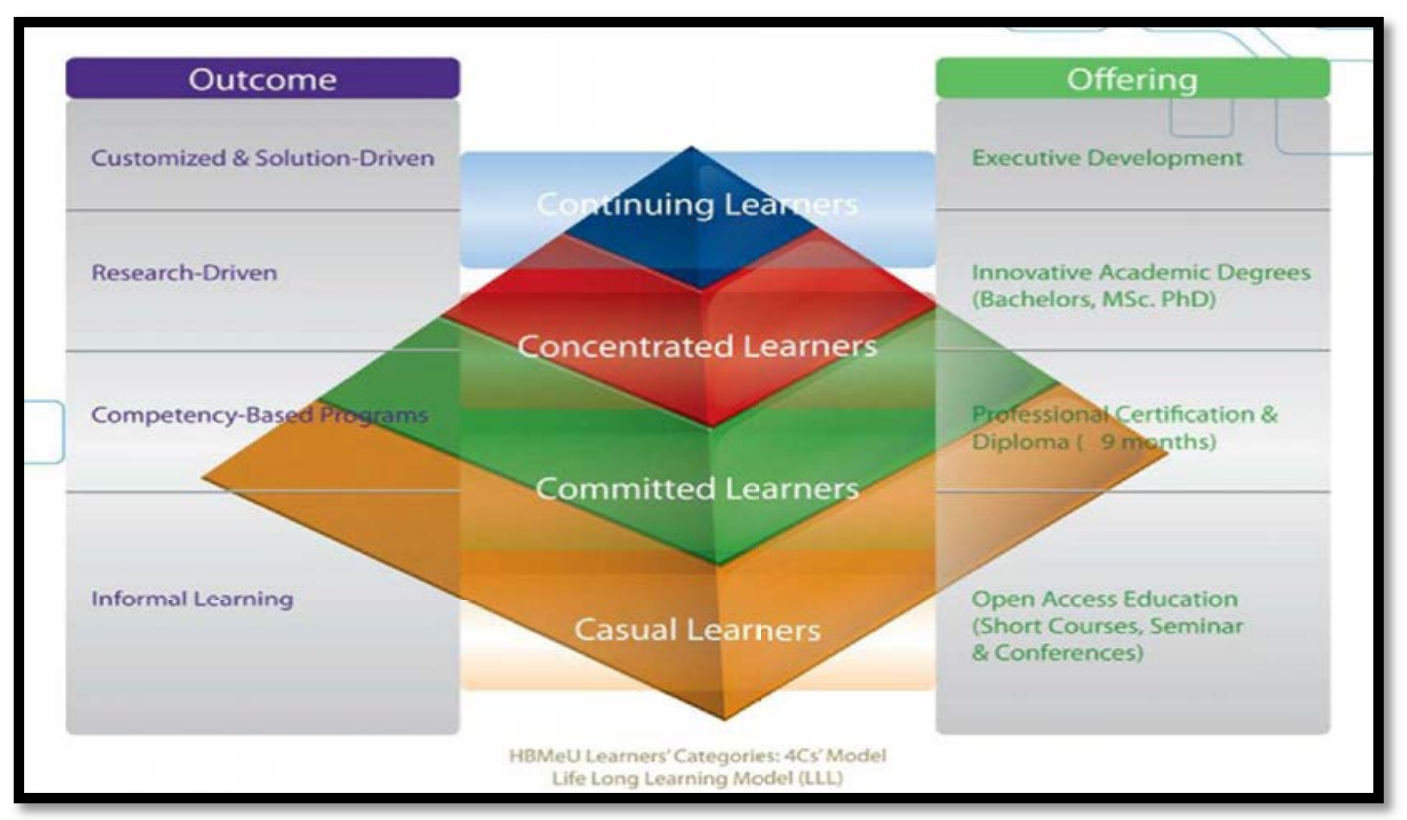

Figure 2. Types of Life Long (Online) Learners 
Promoting Personalized Learning Design: The Role of Online Pedagogical Intervention Hamdy A. Abdelaziz

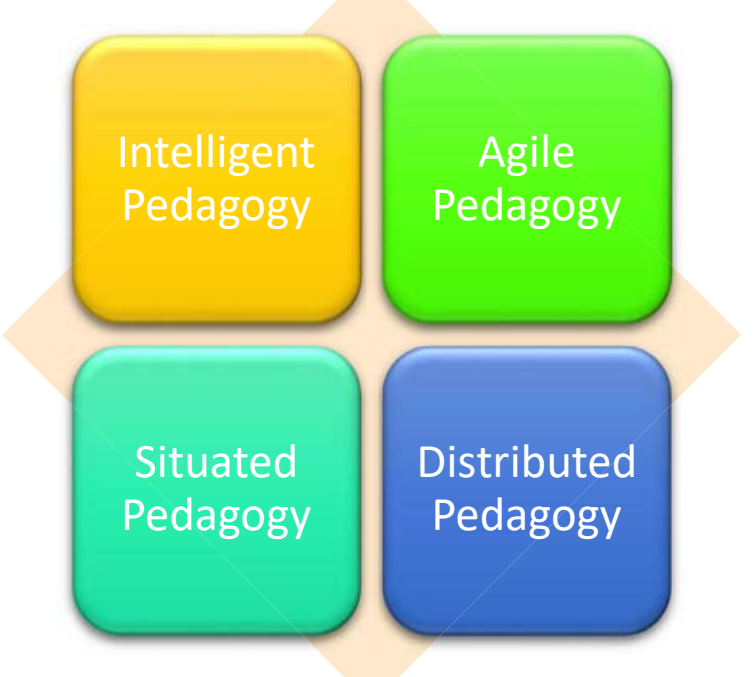

Figure 3. Pedagogical Patterns

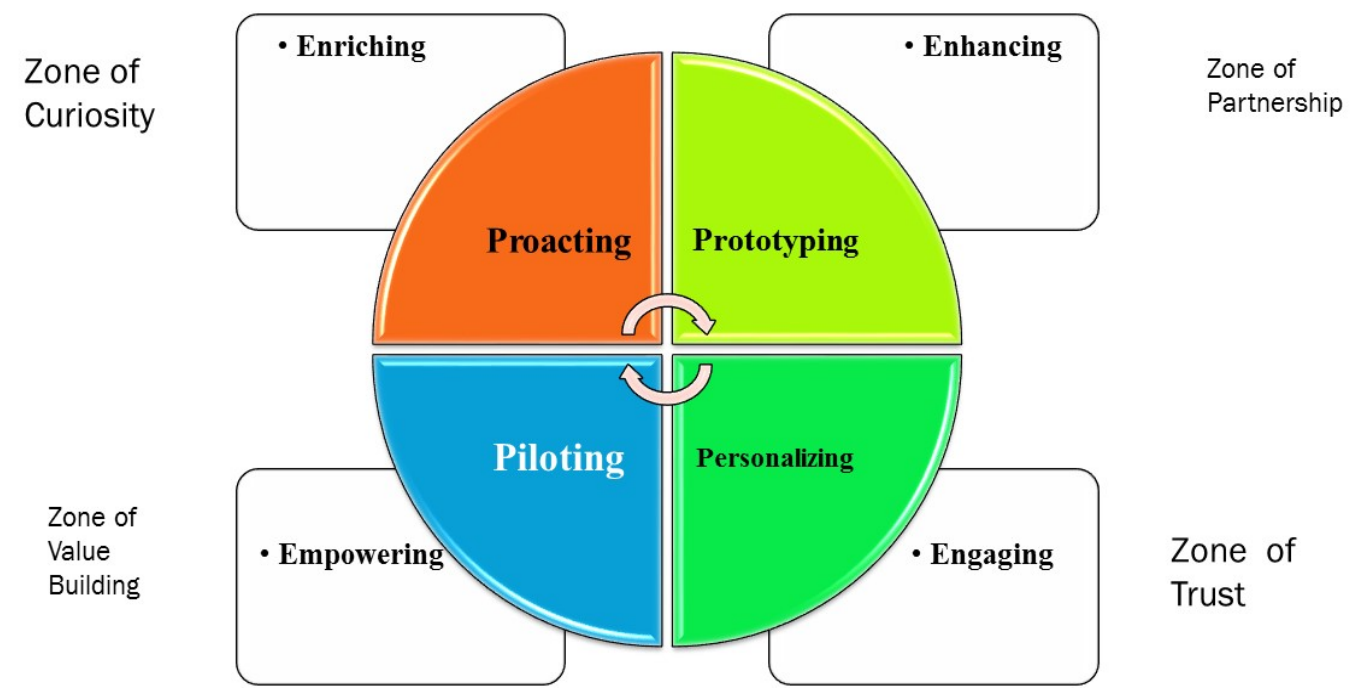

Figure 4. PI Intervention Levels 


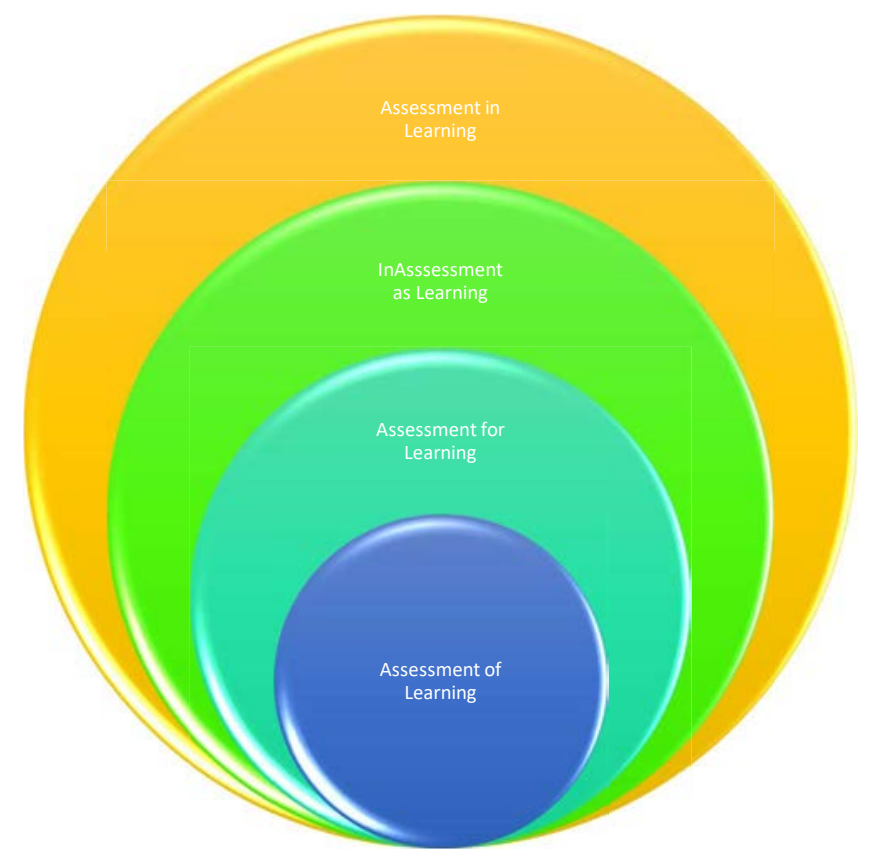

Figure 5. Assessment Frames

\section{Mapping Learning Principles with PI Model}

The following is an example to link one of the ten previously mentioned learning principles. "Strong and profound knowledge is gained through a networking process of collective mind and collective efficacy." To engineer the pedagogical intervention of this principle, online instructor should support his leaners (any level of learners) to interact with factual, conceptual, procedural and metacognitive knowledge by creating personal and group or collective learning spaces. These e-spaces are supported by Intelligent Pedagogy (e.g. creative utilization of emerging technologies), or by Agile pedagogy (e.g. facilitating personalization and flexibility of learning pathways), or by Distributed pedagogy (e.g. increase the focus on learning task and problem design and analysis), or by Situated pedagogy (e.g. increase the focus on context - big ideas or work-related issues/examples). Applying these four pedagogical levels needs sequential and parallel treatment from online mediator to enrich, enhance, engage and empower online learners' collective efficacy.

To validate this model, the researcher applied it on a sample of concentrated leaners $(n=23)$ studying a blended master degree in education registered in fall 2016 at Hamdan Bin Mohammed Smart University. The following are four samples of leaners' reflection about this model (Learners' reflection space (Sawti), HBMSU, 2016-17). 


\section{Search results}

Im writing here to express my deep interest to continue my study in this UNI that considered as advanced and prestigious educational edifice with various study disciplines and quality of learn; aiming also to expand knowledge and gain meaningful taste in my work, study as well as obtaining a gratifying social role in the future. For the class of Research Methods with Professor Dr Hamdy Abdulaziz I began my study and would like to point out some significant benefits I myself acquired. First of all, I found his work delivered properly certainly when it comes to th research's importance, role, value and consideration. Secondly, his GREAT patience with our different level of understandings as well as constant cooperation to clarifying any misconception information. Thirdly, many and many and many terminologies, concepts, aspects regarding research methods have eventually clarified! These outcomes from this class made me very happy since I really was so enthusiastic to seek research tools and techniques' comprehend. On the other hand, I just have one tiny criticism about the tasks given to us weekly, very intensive and long where most of us do not have the time to complete them, so kindly please minimize them in order to provide comfort for all students Thank you Professor for your efforts in this semester ... Kindest regards, Maneh Alahbabi

Take me there $\rightarrow$

\section{Search results}

The scale of measurement is very interesting topic as it assists the reasercher cuning the data collection, there are fou: types of S.M which are nominal, ordinal, interval and ratio to make it more easy to remember call it NOR. Last semester i was enro.led in a course cal.ed 'Research method in Eeducation LEAD600' with Prof. Qhabdelaziz, we leaned step by step how to conduct and write a good research paper. He guided, instuct and taught us many important and useful steps that i refer to whenever i start writing my assignment papers. Its real.y very important to take RESEARCH METHCD training prog or courses before you enro. in any other cou'ses, as it important for the masters degree students to know step by step how to write and submit a high quality pape: Thank you

\section{Take me there $\rightarrow$}

\section{My learning moments (Week 1)}

1. The first L.M $i$ learned is to write down a learning moments in any kind of field. Whether if $i$ am attending a ecture, reading a book, talking to my mother, watching a tv show. I will start to write down anything new $i$ earn and collect all the information in one notebook and by the end of each week $i$ will go through my notes share and discuss it with my friends, family... etc.

2. It was so useful to know about the DIKW Hierarchy which stands for Data, information, knowledge and Nisdom. On the same day, i went back home ad read a lot about DIKW pyramid and the relation between sach element in the pyramid.

Thank you Professor Hamdy Abdullaziz

Hind Almazmi 20010221 


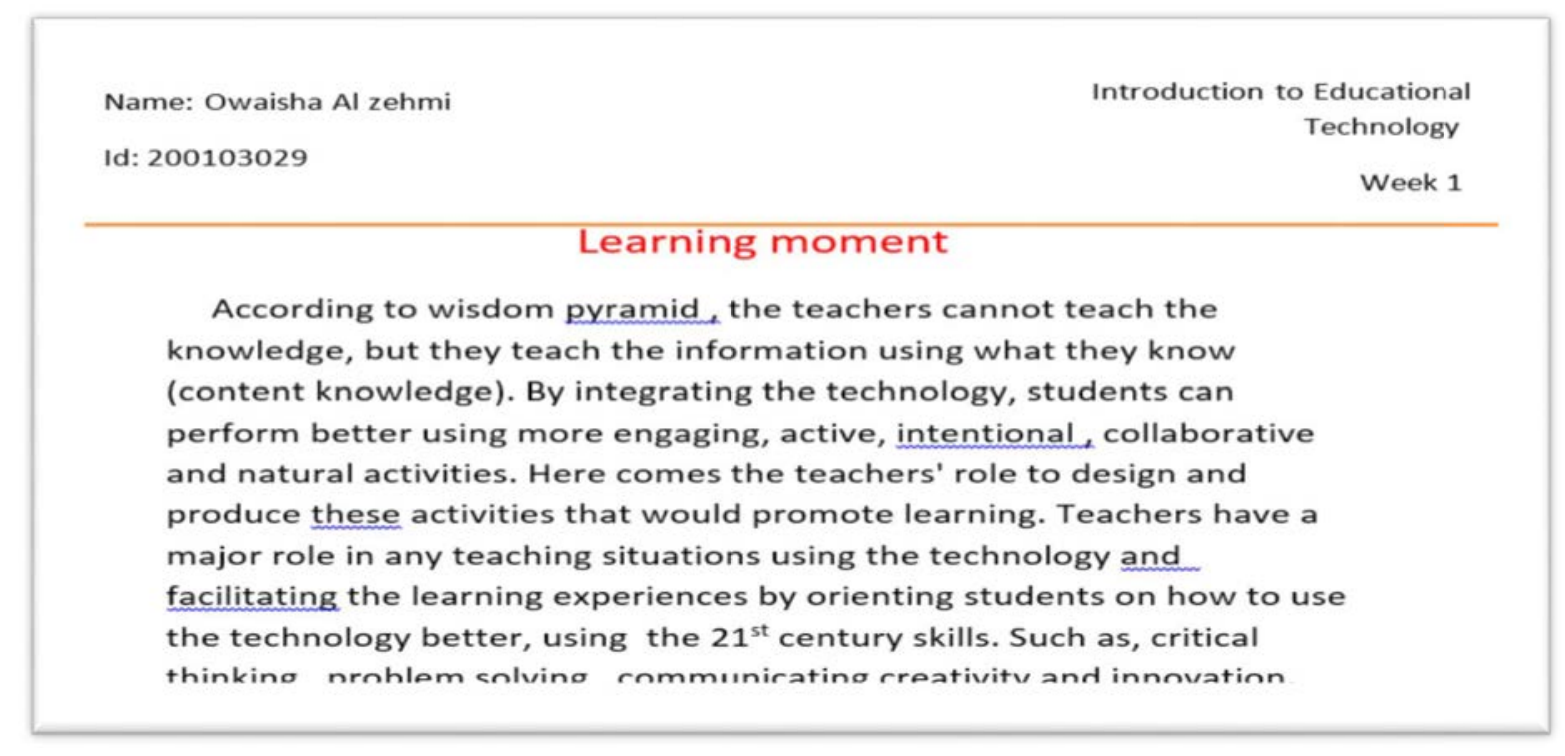

\section{Conclusion and Recommendations}

Online education in the fourth industrial revolution is facing a paradigm shift. This shift is very impacted by the advancement of learning technologies and learners' emergent attributes. Online learning technology, design and delivery modes are maximizing and optimizing the potential chances of personalized, customized, and adaptive learning. In the near future, online education and training models, programs, strategies and platforms will focus on developing human capital and learners' disposition and attribute to foster an innovationdriven culture of learning for impact. Without a plan for transformative pedagogical design, smart technology often remains ancillary to personalized learning. In the current theoretical paper, the author presented a grounded perspective that could be used to increase the level and quality of online pedagogical design and intervention to promote penalized learning. This perspective was guided by ten emergent disruptive learning principles of the third renaissance learning paradigm that impact online pedagogical engineering, management and intervention for personalized learning design.

Online pedagogical intervention has four major lenses that reflect types of learners, pedagogical approaches, levels of intervention, and assessment frames. Pedagogical intervention in online learning context is a cubic and grounded/interacted perspective and focuses on four metaphoric lenses: (a) types of learners (4Cs): Casual, Committed, Concentrated and Continuing; (b) pedagogical levels (4Ps): Intelligent, Agile, Distributed and Situated Pedagogy; (c) intervention levels (4Es): Enriching, Enhancing, Engaging and Empowering; and (d) online assessment frames (4As): assessment of learning, assessment for learning, assessment as learning, and assessment in learning.

As per the theoretical perspective presented in this paper, and as per the indicators that were collected from a piloting process, the author recommends the following highlights to be considered from online learning providers, instructors and practitioners: 
- A shift from the concept of online teaching to online pedagogical engineer and intervention is highly demanded to promote personalized learning design and assessment.

- Assuring the quality of online learning needs a focus on humans' capital (online instructors, facilitators or pedagogists) and their role to apply and evaluate effective pedagogical practices, not only best practices.

- Online learning programs' providers need to invest on the concept of "Online Pedagogist" to make a transformation toward online pedagogical design, engineering, management, and intervention. The outcome of this paper could be used as a focal and starting point to mobilize this recommendation.

\section{References}

Abdelaziz, H. A. (2012). D4 S4: A Four Dimensions Instructional Strategy for Web-based Learning. Turkish Online Journal of Distance Education, 13(4), 220-235.

Abdelaziz, H. A. (2013a). Bridges of Trust: Towards a Constructive Model for Assessing Webbased and Electronic Learning. International Journal of Technology Diffusion, 4(2), 53-70.

Abdelaziz, H. A. (2013b). STEPP: A Grounded Model to Assure the Quality of Instructional Activities in e-learning Environments. Turkish Online Journal of Distance Education (TOJDE), 14(3), 56-77.

Abdelaziz, H. A. (2014a). Creative Design of Interactive eLearning Activities and Materials (IEAM): A Psycho-Pedagogical Model. International Journal of Technology Diffusion, 5(4), 14-34.

Abdelaziz, H. A. (2014b). Immersive learning design (ILD): A new model to assure the quality of learning through flipped classrooms. Open Journal of Social Sciences, 2, 212-223. http://dx.doi.org/10.4236/jss.2014.211027

Abdelaziz, H. A. (2015, November). The Echo of Value: A Suggested Model to Build a Culture of Personalized Learning through Digital Media. Paper presented at The D4Learning 2015, International Conference on Innovations with Digital Learning for Inclusion (d4l) in Aalborg, Denmark, November 17-20, 2015.

Al-Aawar, M. (2009). 4Cs model for smart learning in higher education. Retrieved from http://www.hbmsu.ac.ae

Ally, M. (2008). Foundations of educational theory for online learning. In T. Anderson (Ed.), The Theory and Practice of Online Learning (2 ${ }^{\text {nd }}$ ed., pp. 15-44). AU Press.

Anderson, T. (2008). Teaching in an online learning context. In T. Anderson (Ed.), The Theory and Practice of Online Learning ( $2^{\text {nd }}$ ed., pp. 273-294). AU Press.

Burke, P. J., \& Crozier, G. (2013). Teaching inclusively: Changing pedagogical spaces ( $2^{\text {nd }}$ ed.). University of Roehampton. Retrieved from https://www.newcastle.edu.au/_data/assets/pdf_file/0004/305968/UN001_Teaching_Incl usively_Resource_Pack_Online.pdf 
Chonody, J. M. (2015). Addressing ageism in students: a systematic review of the pedagogical intervention literature. Educational Gerontology, 41, 859-887. doi: 10.1080/03601277.2015.1059139

Guardia, L., Witthaus, G., Rodriguez, B., \& Campillo, C. (2016). Next generation pedagogy: IDEAS for online and blended higher education. University of Oberta de Catalunya. Retrieved from http://openaccess.uoc.edu/webapps/o2/bitstream/10609/51441/1/Next_Generation_Pedag ogy.pdf

Gustafson, K. L., \& Branch, R. M. (2002). Survey of instructional development models (4 ${ }^{\text {th }}$ ed.). New York: Syracuse University. ERIC Clearinghouse on Information \& Technology.

Lockyer, L., Heathcote, E., \& Dawson, S. (2013). Informing pedagogical action: Aligning learning analytics with learning design. American Behavioural Scientist, 57(10), 14391459. https://doi.org/10.1177/0002764213479367

Mekala, S., Shantha, S., \& Ponmani, M. (2017). The role of pedagogical intervention in improving the oral proficiency of engineering students. The IUP Journal of Soft Skills, 11(3), 7-28.

Ramdass, K., \& Masithulela, F. (2016). Comparative analysis of pedagogical strategies across disciplines in open distance learning at Unisia. International Review of Research in Open and Distributed Learning, 17(2), 1-18. http://dx.doi.org/10.19173/irrodl.v17i2.2402

Robinson, K. (2011). Out of our minds: Learning to be creative ( $2^{\text {nd }}$ ed.). UK: Capstone Publishing Ltd.

Saville, B., Zinn, T., \& Elliott, M. (2005). Interteaching versus traditional methods of instruction: A preliminary analysis. Teaching of Psychology, 32(3), 161-163.

Shum, S. B., \& Ferguson, R. (2012). Social learning analytics. Educational Technology \& Society, 15(3), 3-26.

Silva, J. (2013). Understanding the construction of the teaching discourse in an on-line environment. International Education Studies, 6(3), 143-155.

http://dx.doi.org/10.5539/ies.v6n3p143

Tegos, S., Demetriadis, S., Papadopoulos, P., \& Weinberger, A. (2016). Conversational agents for academically productive talk: A comparison of directed and undirected agent interventions. International Journal of Computer-Supported Collaborative Learning, 11(4), 417-440. http://dx.doi.org/10.1007/s11412-016-9246-2

Westberry, N., \& Fanken, M. (2015). Pedagogical distance: Explaining misalignment in student-driven online learning activities using Activity Theory. Teaching in Higher Education, 20(3), 300-312. http://dx.doi.org/10.1080/13562517.2014.1002393

Wise, A. F. (2014). Designing pedagogical interventions to support student use of learning analytics. Indianapolis, IN, USA: ACM. http://dx.doi.org/10.1145/2567574.2567588 
Wright, R., \& Collins, D. (2007). Developing pedagogical tools for intervention: Approach, methodology, and an experimental framework. Proceedings of the $30^{\text {th }}$ Annual Conference of the Mathematics Education Research Group of Australasia, J. Watson \& K. Beswick (Eds), 843-852. MERGA Inc.

Zhu, Z. T., Yu, M. H., \& Riezebos, P. (2016). A research framework of smart education. Smart Learning Environments, 3(4), March 2016. http://dx.doi.org/10.1186/s40561-016-0026-2 